\section{Food storage temperatures monitored at retail}

\author{
Nicola Costanzo, ${ }^{1}$ Eleonora Sarno, ${ }^{2,3}$ \\ Salvatore Ferrante, 4 \\ Adriano M.L. Santoro ${ }^{2}$ \\ 1Dipartimento di Scienze della Salute, \\ Università Magna Græcia di Catanzaro, \\ Italy; ${ }^{2}$ Dipartimento di Scienze \\ Zootecniche e Ispezione degli Alimenti di \\ Origine Animale, Università degli Studi di \\ Napoli Federico II, Italy; ${ }^{3}$ Institut für \\ Lebensmittelsicherheit und Hygiene der \\ Universität Zürich, Switzerland; ${ }^{4}$ Medico \\ Veterinario, libero professionista, Napoli, \\ Italy
}

\section{Abstract}

Aim of the present work is to report data concerning the maintenance of the cold chain by retail food business operators. A total of 401 refrigerators and 105 freezers from 112 retails (big, medium, small size) were monitored for display temperatures. In addition, the surface temperature of 341 stored food products was recorded. Storage temperatures were respected in the majority of retail markets, with the exception of small retails, where cold chain was not respected. Among all food samples, yogurt was stored at temperature higher than law limits. Our findings show that retailers, in particular those from small markets, are not always familiar with cold chain maintenance. In our opinion, much more attention should be paid in keeping food at cold temperature in order to ensure food safety.

\section{Introduzione}

Il rispetto della catena del freddo, compreso nel corso dello stoccaggio, durante la commercializzazione ed esposizione, e immediatamente prima dell'acquisto da parte del consumatore, è alla base di un corretto mantenimento dello standard qualitativo e sanitario dei prodotti alimentari. La corretta applicazione della catena del freddo necessita, quindi, di un costante monitoraggio anche durante la vendita al minuto (Reg. CE 853/2004; Commissione Europea, 2004). Scopo del presente lavoro è stato quello di monitorare le temperature di refrigerazione e surgelazione durante la vendita al dettaglio di alcuni esercizi commerciali al fine di verificare il mantenimento della catena del freddo da parte degli operatori del settore alimentare secondo i regolamenti vigenti in materia di igiene e sicurezza alimentare.

\section{Case Report}

Nel corso dell'anno 2011 nella città di Napoli, sono stati selezionati per la presente ricerca 112 esercizi commerciali di vendita al dettaglio di prodotti alimentari di origine animale classificati, in funzione del DL 114/98 (Repubblica Italiana, 1998), in grandi strutture di vendita (n. 2), medie strutture di vendita (n. 25) ed esercizi di vicinato (n. 85). In ogni esercizio si è proceduto a: i) registrazione dei frigoriferi e dei congelatori; ii) lettura della temperatura delle attrezzature frigorifere e dei congelatori indicate dai relativi display e misurazione della temperatura ambientale al loro interno mediante termometri elettronici (HI93510N; Hanna Instruments, Padova, Italia) tarati e posizionati in corrispondenza di quelli propri delle attrezzature stesse; iii) misurazione, mediante termometri elettronici tarati, della temperatura superficiale delle confezioni di alimenti refrigerati e surgelati posti negli appostiti banchi espositivi.

Il numero totale di attrezzature considerate per il presente lavoro è stato pari a 401 frigoriferi e 105 congelatori per un totale di 506 .

Le tipologie di confezioni alimentari analizzate, per un totale di 341 campioni, erano così suddivise: carni rosse (n. 104) e bianche (n. 77), latte pastorizzato (n. 51), yogurt (n. 35) e surgelati (n. 74).

Display/termometri erano assenti o non funzionanti in 62 (12,3\%) (37 frigoriferi e 25 congelatori) delle 506 strutture frigorifere monitorate. Tale situazione è stata riscontrata solo negli esercizi di vicinato.

Le temperature indicate dai display e quelle misurate mediante termometro elettronico sono riportate nelle Tabelle 1 e 2 , rispettivamente per frigoriferi e congelatori. Le temperature non conformi, invece, sono riportate nella Tabella 3 .

\section{Discussione}

I dati raccolti evidenziano che è necessario prestare attenzione al monitoraggio delle temperature di refrigerazione e di surgelazione. In alcuni esercizi di vicinato i display erano talora assenti o non funzionanti, non consentendo in tal modo il controllo della temperatura. Differenze sono state riscontrate tra le temperature indicate dai display e quelle rilevate mediante misurazione con termometri tarati. La temperatura misurata è risultata mediamente più elevata rispetto a quella mostrata dal display di $1^{\circ} \mathrm{C}$ (Tabella 1) nei frigoriferi di $2{ }^{\circ} \mathrm{C}$ nei congelatori (Tabella 2). Queste evidenze sono state osservate maggiormente negli esercizi di vicinato nonché in qualche esercizio di media grandezza. Nessuna differenza tra
Correspondence: Nicola Costanzo, Dipartimento di Scienze della Salute, Università Magna Græcia di Catanzaro, viale Europa, 88100 Germaneto, Italy.

Tel. +39.366.6582808 - Fax: +39.081 .19972759 .

E-mail: costanzo.nic@unicz.it

Key words: Cold chain, Retails, Food safety.

Conflict of interests: the authors declare no potential conflict of interests.

Received for publication: 15 January 2013. Revision received: 29 March 2013.

Accepted for publication: 2 April 2013.

This work is licensed under a Creative Commons Attribution 3.0 License (by-nc 3.0).

(C) Copyright N. Costanzo et al., 2013 Licensee PAGEPress, Italy

Italian Journal of Food Safety 2013; 2:e14 doi:10.4081/ijfs.2013.e14

la temperatura riportata dai display e quella misurata è stata evidenziata in esercizi di grandi dimensioni. Non molti dati provengono dalla letteratura riguardo tali misurazioni, ciononostante i dati esposti nel presente lavoro sembrano in linea con precedenti ricerche condotte in altri Paesi. Derens et al. (2006), in uno studio condotto in Francia allo scopo di monitorare la catena del freddo nei banchi vendita self-service di alimenti in grandi e medi punti vendita, hanno evidenziato che il $3 \%$ degli alimenti refrigerati superava le temperature di refrigerazione consentite per legge. Altri autori, in ricerche condotte rispettivamente in Slovenia e Grecia (Likar e Jevskin, 2006; Koutsoumanis e Angelidis, 2007), riferiscono di temperature medie di $1^{\circ} \mathrm{C}$ più alte rispetto a quelle rilevate nel presente lavoro.

Per quanto riguarda la misurazione della temperatura sulla superficie delle confezioni (Tabella 3) è da rilevare che per i campioni di carni rosse, ad eccezione di un caso relativo ad un medio esercizio commerciale, le temperature sono risultate sempre entro il limite di $+7^{\circ} \mathrm{C}$ previsto dal Reg. CE 853/2004 (Commissione Europea, 2004). Nei piccoli esercizi, circa il $20 \%$ dei campioni di carne bianca eccedeva le temperature previste (Commissione Europea, 2004). Circa il 35\% dei campioni di latte pastorizzato era stoccato a una temperatura superiore a $+6^{\circ} \mathrm{C}$, soprattutto negli esercizi di vicinato.

L'abuso di temperatura in questo prodotto comporta diversi problemi, tra cui una precoce comparsa di odori e sapori anormali tipici dei microrganismi di spoilage quali Pseudomonas spp. (Hayes et al., 2002; Ternström et al., 1993). Nei piccoli e medi esercizi di vendita, $i$ campioni di yogurt hanno mostrato una tempe- 
ratura di stoccaggio superiore a quella prevista dal produttore. Variazioni di temperatura in questi prodotti, per quanto ampiamente tollerate durante la fase del trasporto (Allegato $\mathrm{C}$ Parte II DPR 327/80 come sostituito da D.M. 01/04/88 n 178; Repubblica Italiana, 1980), sono da evitare soprattutto durante la fase di stoccaggio in quanto incidono sulla shelf life e sulle caratteristiche organolettiche dei prodotti, nonché sulla perdita di vitalità di batteri probiotici in esso contenuto (Scharl et al., 2011). Variazioni di temperatura sono state inoltre osservate per i campioni di surgelati, dove tuttavia una fluttuazione $\mathrm{di}+3^{\circ} \mathrm{C}$ è tollerabile (DL n. 110 del 27.1.92; Repubblica Italiana, 1992).

\section{Conclusioni}

È nostra opinione che un più rigido rispetto della catena del freddo debba essere osservato da parte degli operatori del settore alimentare soprattutto nei piccoli punti vendita.

Realizzare una catena del freddo controllata

Tabella 1. Registrazione delle temperature indicate dai display delle attrezzature frigorifere e quelle rilevate mediante termometri tarati posizionati in corrispondenza di quelli propri delle attrezzature stesse.

\begin{tabular}{lccccccc}
$\begin{array}{l}\text { Frigoriferi } \\
\text { (n=364) }\end{array}$ & $\mathrm{T}$ & $\mathrm{T}$ & $\mathrm{T}$ & $\mathrm{T} \leq 4^{\circ} \mathrm{C}$ & $4^{\circ} \mathrm{C}<\mathrm{T}<6^{\circ} \mathrm{C}$ & $6^{\circ} \mathrm{C}<\mathrm{T}<8^{\circ} \mathrm{C}$ & $\mathrm{T}>8^{\circ} \mathrm{C}$ \\
T display & -4 & massima $\left({ }^{\circ} \mathrm{C}\right)$ & $\operatorname{media}\left({ }^{\circ} \mathrm{C}\right)$ & & & \\
T rilevata & $-4,4$ & 14,7 & $2,88( \pm 0,23)$ & 249 & 87 & 19 \\
\hline
\end{tabular}

T, temperatura.

Tabella 2. Registrazione delle temperature indicate dai display dei congelatori e quelle rilevate mediante termometri tarati posizionati in corrispondenza di quelli propri delle attrezzature stesse.

\begin{tabular}{lcccccccc}
$\begin{array}{l}\text { Congelatori } \\
(\mathrm{n}=80)\end{array}$ & minima $\left({ }^{\circ} \mathrm{C}\right)$ & massima $\left({ }^{\circ} \mathrm{C}\right)$ & media $\left({ }^{\circ} \mathrm{C}\right)$ & $\mathrm{T} \leq-30^{\circ} \mathrm{C}$ & $\mathrm{T} \leq-26^{\circ} \mathrm{C}$ & $\mathrm{T} \leq-22^{\circ} \mathrm{C}$ & $\mathrm{T} \leq-18^{\circ} \mathrm{C}$ & $\mathrm{T}>-18{ }^{\circ} \mathrm{C}$ \\
T display & -35 & -16 & -23 & 9 & 7 & 24 & 35 \\
Trilevata & -30 & -14 & -21 & 0 & 8 & 14 & 36 \\
\hline
\end{tabular}

$\mathrm{T}$, temperatura.

Tabella 3. Confezioni di alimenti con temperature non conformi a quelle consentite per legge.

\begin{tabular}{|c|c|c|c|c|c|c|c|}
\hline $\begin{array}{l}\text { Prodotti } \\
\text { conferionati }\end{array}$ & $\begin{array}{l}\mathrm{T} \\
\text { consentita }\end{array}$ & $\begin{array}{l}\text { Riferimento } \\
\text { normativo }\end{array}$ & $\begin{array}{l}\text { Provenienza } \\
\text { (n. strutture) }\end{array}$ & $\begin{array}{l}\text { \% sul numero } \\
\text { di difformità }\end{array}$ & $\begin{array}{l}\text { Campioni } \\
\text { (n. totale) }\end{array}$ & $\begin{array}{c}\text { Campioni } \\
\text { difformi (n.) }\end{array}$ & $\begin{array}{c}\text { Difformità } \\
(\%)\end{array}$ \\
\hline Carni rosse & $+7^{\circ} \mathrm{C}$ & $\begin{array}{l}\text { Reg. CE 853/04 } \\
\text { (Commissione Europea, } \\
\text { 2004) }\end{array}$ & $\begin{array}{l}\text { Esercizi di vicinato (0) } \\
\text { Medie strutture }(1) \\
\text { Grandi strutture }(0)\end{array}$ & $\begin{array}{c}0 \\
100 \\
0\end{array}$ & 104 & 1 & 0,96 \\
\hline Carni bianche & $+4^{\circ} \mathrm{C}$ & $\begin{array}{l}\text { Reg. CE 853/04 } \\
\text { (Commissione Europea, } \\
\text { 2004) }\end{array}$ & $\begin{array}{l}\text { Esercizi di vicinato (9) } \\
\text { Medie strutture }(6) \\
\text { Grandi strutture }(0)\end{array}$ & $\begin{array}{c}66,6 \\
33,3 \\
0 \\
\end{array}$ & 77 & 15 & 19,5 \\
\hline Latte pastorizzato & $1^{\circ} \mathrm{C}<\mathrm{T}<6^{\circ} \mathrm{C}$ & $\begin{array}{l}\text { Art. } 5 \text { L. } 3.5 .89 \text { n. } 169 \\
\text { (Repubblica Italiana, 1989) }\end{array}$ & $\begin{array}{l}\text { Esercizi di vicinato (14) } \\
\text { Medie strutture (4) } \\
\text { Grandi strutture (0) }\end{array}$ & $\begin{array}{c}61,3 \\
34,1 \\
0\end{array}$ & 51 & 18 & 35,3 \\
\hline Yogurt & $+4^{\circ} \mathrm{C}$ & Indicata dal produttore & $\begin{array}{l}\text { Esercizi di vicinato (11) } \\
\text { Medie strutture (16) } \\
\text { Grandi strutture (3) }\end{array}$ & $\begin{array}{c}36,7 \\
53,3 \\
100 \\
\end{array}$ & 35 & 30 & 85,7 \\
\hline \multirow[t]{3}{*}{ Surgelati } & $<-18^{\circ} \mathrm{C}$ & $\begin{array}{l}\text { Riforma dell'articolo 4, } \\
\text { comma 4, della legge } \\
15 \text { marzo 1997, n. } 59, \\
\text { DL 114/98 (Repubblica } \\
\text { Italiana, 1998) }\end{array}$ & Esercizi di vicinato (10) & 66,6 & & & \\
\hline & \multirow{2}{*}{$\begin{array}{l}\text { Fluttazioni } \\
\text { possibili } \\
\text { fino a }-15^{\circ} \mathrm{C}\end{array}$} & \multirow{2}{*}{$\begin{array}{l}\text { DL } 27.01 .92 \text { n. } 110 \\
\text { (Repubblica Italiana, 1992) }\end{array}$} & Medie strutture (3) & 20 & \multirow[t]{2}{*}{74} & \multirow[t]{2}{*}{15} & \multirow[t]{2}{*}{20,3} \\
\hline & & & Grandi strutture (2) & 13,4 & & & \\
\hline
\end{tabular}


fino alla vendita al consumatore finale rappresenta, per le aziende di produzione che trattano prodotti a temperatura controllata, un'esigenza per garantire l'integrità del prodotto. Disporre di apparecchi adatti a rilevare e registrare nel tempo la temperatura diventa fondamentale per poter garantire il rispetto delle temperature di refrigerazione. Recentemente sono stati impiegati come ausili dei dispositivi di monitoraggio della storia termica [time temperature integrators (TTI)] configurati sotto forma di etichette adese alla confezione del prodotto e strutturate in modo tale da manifestare una variazione cromatica proporzionale all'estensione del tempo temperatura. Questo monitoraggio consentirebbe al produttore di avere un importante ritorno di immagine e di fiducia, e al consumatore di acquistare prodotti sicuri. Infine esso obbligherebbe il rivenditore ad un più stretto controllo sulle temperature all'interno degli esercizi commerciali.

\section{Bibliografia}

Commissione Europea, 2004. Regolamento del parlamento europeo e del consiglio del 29 aprile 2004 che stabilisce norme specifiche in materia di igiene per gli alimenti di origine animale, 853/2004/CE. In: Gazzetta Ufficiale, L 139/55, 30/04/2004.

Derens E, Palagos B, Guilpart J, 2006. The cold chain of chilled products under supervision in France. IUFoST 13th World Congress of Food Sciences Technology, 2006 Sept 17-21, Nantes, France.

Hayes W, White CH, Drake MA, 2002. Sensory aroma characteristics of milk spoilage by Pseudomonas Species. J Food Sci 67:448-54.

Koutsoumanis K, Angelidis AS, 2007. Probabilistic modeling approach for evaluating the compliance of ready-to-eat foods with new european union safety criteria for Listeria monocytogenes. Appl Environ Microbiol 73:4996-5004.

Likar K, Jevsnik M, 2006. Cold chain maintaining in food trade. Food Control 17:108-13.

Repubblica Italiana, 1980. Decreto del Presidente della Repubblica n. 327 del 26/03/1980. Regolamento di esecuzione della Legge 30 aprile 1962, n. 283, e successive modificazioni, in materia di disciplina igienica della produzione e della ven- dita delle sostanze alimentari e delle bevande. In: Gazzetta Ufficiale no. 193, 16/07/1980.

Repubblica Italiana, 1989. Disciplina del trattamento e della commercializzazione del latte alimentare vaccino, Legge 03-051989, n. 169. In: Gazzetta Ufficiale n. 108, 11-05-1989.

Repubblica Italiana, 1992. Attuazione della direttiva no. 89/108/CEE in materia di alimenti surgelati destinati all'alimentazione umana, DL 39/1992. In: Gazzetta Ufficiale no. 110, 27/01/1992.

Repubblica Italiana, 1998. Riforma della disciplina relativa al settore del commercio, a norma dell'articolo 4, comma 4, della legge 15 marzo 1997, n. 59, DL 114/98. In: Gazzetta Ufficiale no. 95, 24/04/1998.

Scharl M, Geisel S, Vavricka SR, Rogler G, 2011. Dying in yoghurt: the number of living bacteria in probiotic yoghurt decreases under exposure to room temperature. Digestion 83:13-7.

Ternström A, Lindberg AM, Molin G, 1993. Classification of the spoilage flora of raw and pasteurized bovine milk, with special reference to Pseudomonas and Bacillus. J Appl Bacteriol 75:25-34. 